\title{
Tattooing and psoriasis: demographics, motivations and attitudes, complications, and impact on body image in a series of 90 Finnish patients
}

\author{
Nicolas Kluger ${ }^{1 凶}$
}

\begin{abstract}
Introduction: Koebner phenomenon (KP) affects up to a third of patients with psoriasis and can occur on tattoos. Little is known about the extent of tattooing and its consequences among psoriatic patients. A survey was conducted to determine the demographics, motivations and attitudes towards tattoos, and complications among tattooed patients with psoriasis, and the impact on their body image.

Methods: Ninety Finnish patients completed an internet self-reported questionnaire in June 2016.

Results: Fifty-two percent (48/90) had one tattoo or more (mean number of three tattoos, range 1-20). They were younger than non-tattooed patients $(p=0.001)$. Of these, $27.6 \%$ experienced a KP on their tattoos from 1 week to 15 to 20 years after tattooing. Among those, $30 \%$ reported an acute flare-up of psoriasis within the first weeks after tattooing. They were more likely to have a history of KP. Less than $7 \%$ reported a psoriasis flare-up on another part of the body after tattooing. Eighty-two percent stated that their tattoo(s) had a positive effect on their body image.

Conclusion: KP on tattoos is not particularly frequent in patients with psoriasis. Tattooing has a bolstering effect on body image and should not be a contraindication. However, patients need proper counseling before receiving tattoos.
\end{abstract}

Keywords: tattooing, tattoo, psoriasis, Koebner phenomenon, body image

Received: 1 March 2017 | Returned for modification: 8 April 2017 | Accepted: 16 April 2017

\section{Introduction}

Psoriasis is one of the most common chronic skin conditions and affects approximately $2 \%$ of the general population (1). One of the key features of psoriasis, albeit not specific, is the possible localization of lesions on traumatized areas and scars (also well known as the Koebner phenomenon, KP) (2-5). It occurs in approximately one-quarter (4) to one-third (5) of patients with psoriasis after various traumas and with variable delays from weeks to usually a few years (3). In his initial description of the phenomenon, Heinrich Koebner recorded the specific localization of psoriasis on tattoos $(2,3,5)$. Since then, case reports have been reported since 1940 in the literature (6-20). In addition, an anecdotal case of psoriatic arthritis triggered after tattooing has been recorded (7).

Tattooing can be considered "mainstream" because 10 to $20 \%$ of the general population has one or more tattoos (21). In Finland, $13 \%$ of people 15 to 29 years old are tattooed (22). It is therefore no surprise that patients with psoriasis may be interested in getting tattooed. Patients may seek advice from professional tattooists and dermatologists regarding possible risks during tattooing. For the author, psoriasis is not a strict contraindication for tattooing (23). Current advice to patients includes: i) avoiding getting a tattoo in the case of active psoriasis, ii) knowing that there is a greater risk of KP for patients with a previous history of KP on other scars, and iii) using extra caution when getting a tattoo during immunosuppressive treatment (anti-TNF alpha, etc.) (24).

Overall, there is a lack of knowledge of the extent of this practice among psoriatic patients and the frequency of complications, mainly psoriasis flare-ups and KP. To address these issues, we carried out a national internet survey among Finnish patients with psoriasis and investigated the profile of patients getting tattoos, their motivations and attitudes, and the complications they experienced.

\section{Material and methods}

\section{Design}

The study was performed with the participation of the Finnish Psoriasis Association (Psoriasisliitto ry). A standardized anonymous questionnaire was available on Google Forms. Patients were recruited by placing an advertisement for the study and the internet link to the questionnaire on the social network pages of the association (Facebook and Twitter). The study took place from June 1oth to July 1st, 2016 and the answers were downloaded in an Excel file for analysis. Only the author has access to the data. Participants freely took part in the study and no incentive was given in exchange for participation.

\section{Survey}

The questionnaire was divided into five parts:

1) The author asked about the background of the patient, including demographic data (sex and age), psoriasis (age at onset, psoriasis phenotype, and presence of KP defined by localization of psoriasis on traumatized areas in the past), and the presence of tattoos.

2) Patients without tattoos were invited to explain whether they were planning to get a tattoo in the future and, if not, why.

3) Tattooed patients were asked about the number of tattoos and the conditions under which they received them (Had they had a prior discussion with their doctor before tattooing? Was the tattooist warned of their psoriasis? Did the psoriasis occur on the tattooed area?). The patients were asked to explain the reasons for getting tattoos in their own words. Their answers were analyzed line by line and sorted mainly by using the motiva- 
teional categories published by Wohlrab et al. (25).

4) If psoriasis occurred on the tattoo, various data were collected such as delay after tattooing, limitation of the psoriasis to the tattooed area, extension to other tattoos, and occurrence on the tattoo every time the patient had a psoriasis flare-up.

5) Finally, the author investigated whether tattooing triggered a cutaneous flare-up of psoriasis on the body or an arthritis flare-up, and whether other tattoo complications occurred. To conclude, patients were asked whether they thought that the tattoos had a positive impact on their body image.

\section{Statistical analysis}

A statistical analysis was conducted with SPSS Statistics 22 (IBM). Patients' characteristics are presented as means and standard deviations (SD) for continuous variables and as frequencies and proportions for categorical variables. Patients' characteristics were compared using a chi-square test for categorical variables and t-test for continuous variables. The statistical significance threshold was set at $p<0.05$.

\section{Results}

\section{Features of the total population study}

A total of 90 patients completed the questionnaire with a large overrepresentation of women (80 women, $89 \%$ of the cohort). The mean age was 40.9 years old (15-70; Table 1$)$. The most common forms of psoriasis were scalp psoriasis $(73 \%)$, plaque psoriasis (64\%), and elbow and knee psoriasis (48\%). A KP was reported by $28 \%$ of the patients (Table 2 ). Among the respondents, $52 \%$ (48/90) had one tattoo or more. No response rate could be established in this study due to its design.

Table 1 | Demographics of the respondents.

\begin{tabular}{|c|c|c|c|c|}
\hline Measure & $\begin{array}{c}\text { Total } \\
n=90\end{array}$ & $\begin{array}{l}\text { With tattoos } \\
\qquad n=48\end{array}$ & $\begin{array}{l}\text { Without tattoos } \\
\qquad n=42\end{array}$ & $p<0.05$ \\
\hline Sex ratio:1 (W:M) & $8(80: 10)$ & $11(44: 4)$ & $6(36: 6)$ & NS \\
\hline $\begin{array}{l}\text { Mean age } \\
\text { (years, SD) }\end{array}$ & $40.9(12.4)$ & $37.9(9.0)$ & $44.3(14.7)$ & 0.001 \\
\hline Ranges (years) & $15-70$ & $20-55$ & $15-70$ & \\
\hline $\begin{array}{l}\text { Mean age at onset } \\
\text { of psoriasis } \\
\text { (years, SD) }\end{array}$ & 22.9 (11.1) & $20.7(9.4)$ & $25.2(12.3)$ & NS \\
\hline $\begin{array}{l}\mathrm{M}=\text { Men, } \mathrm{W}=\text { Wome } \\
\text { Table } 2 \mid \text { Psoriasis } \mathrm{p}\end{array}$ & $n$ & Ig to navi & attoos or not. & \\
\hline Type & $\begin{array}{c}\text { Total } \\
\%(n=90)\end{array}$ & $\begin{array}{l}\text { Tattooed } \\
\%(n=48)\end{array}$ & $\begin{array}{c}\text { Non-tattooed } \\
\%(n=42)\end{array}$ & $\begin{array}{c}x^{2} \\
p<0.05\end{array}$ \\
\hline Koebner & $28(25)$ & $21(10)$ & $28(15)$ & NS \\
\hline Plaques & $64(58)$ & $56(27)$ & $74(31)$ & NS \\
\hline Guttata & $33(30)$ & $37(18)$ & $28(12)$ & NS \\
\hline Pustular & $2(2)$ & $0(0)$ & $5(2)$ & NS \\
\hline Folds & $20(18)$ & $17(8)$ & $24(10)$ & NS \\
\hline Nail & $34(31)$ & $33(16)$ & $36(15)$ & NS \\
\hline Scalp & $73(66)$ & $65(31)$ & $83(35)$ & 0.045 \\
\hline Elbows/knees & $48(43)$ & $42(20)$ & $55(23)$ & NS \\
\hline $\begin{array}{l}\text { Palmo-plantar } \\
\text { pustulosis }\end{array}$ & $12(11)$ & $8(4)$ & $17(7)$ & NS \\
\hline Psoriatic arthritis & $37(33)$ & $31(15)$ & $43(18)$ & NS \\
\hline
\end{tabular}

\section{Features of non-tattooed patients with psoriasis}

The non-tattooed group was significantly older than those with tattoos ( $p=0.001$, Table 1$)$. The mean age at onset of psoriasis was higher in this group (25 years old), but at the limit of significance $(p=0.059)$. The majority of them $(68 \%, 28 / 41)$ would never con- sider a tattoo in the future, whereas $15 \%(6 / 41)$ considered it and $17 \%$ (7/41) said they might. Those that did not want any tattoos were older (mean age 49.6 years old \pm 11.8 ) than those that were considering this possibility ("yes" 32.3 years old \pm 14.2 ; "maybe" 30.4 years old \pm 10.3 ).

For those that would never get a tattoo or were not planning on getting one, $57.1 \%(16 / 28)$ were not interested in tattoos, $28.6 \%$ $(8 / 28)$ feared that tattooing would cause a psoriasis flare-up or worsen psoriasis, and $11 \%$ (3/28) feared infection.

Among those that were planning to get a tattoo, one-third (2/6) feared a psoriasis flare-up or worsening. Interestingly, none reported a KP, and 39.3\% (11/28) of those that would not get any tattoo had an isomorphic phenomenon.

\section{Features of tattooed patients with psoriasis}

This group was significantly younger ( $p=0.001$, Table 1$)$. The mean number of tattoos was $3 \pm 3.26$ (range 1 to 20 tattoos). One patient had only permanent make-up on the eyebrows. Forty-one patients stated in their own terms the reasons or the motivations that compelled them to get one or more tattoos (Table 3). The three main motivational categories were beauty / body embellishment, expression of personal narratives, and individuality. Two patients expressed in their own words what was interpreted as an attempt to regain control over their skin condition.

The prevalence of KP was lower among patients with tattoos, but without reaching significance $(p>0.05)$. Only the difference in the prevalence of scalp psoriasis was statistically significant between the groups (Table 1).

Only 8.5\% (4/47) discussed their wish to get a tattoo with their doctor, whereas $43.5 \%$ (20/46) warned their tattooist about their psoriasis. At the time of tattooing, almost half of the patients $(48.9 \%, 22 / 45)$ were not using any treatment and 46.7\% (21/45) were using local treatment such as corticosteroids or calcipotriol. Four patients were either receiving methotrexate $(6.7 \% 3 / 45)$ or a biologic $(2.2 \%, 1 / 45)$ during tattooing. No one used cyclosporine or acitretine. One patient was receiving phototherapy $(2.2 \%)$.

Table 3 | Psoriasis phenotype according to having tattoos or not.

\begin{tabular}{lc}
\hline Reason & Percent \\
\hline Beauty, body embellishment, and fashion & 41.5 \\
Personal narratives & 24 \\
Individuality & 17 \\
Souvenirs & 17 \\
Wanted a tattoo (no further explanation) & 12 \\
Group affiliations and commitment & 10 \\
$\quad$ Family & 7 \\
Religion & 3 \\
Spirituality & 3 \\
Miscellaneous & 7 \\
\hline
\end{tabular}

${ }^{\star}$ The total is greater than $100 \%$ because the analysis of the response allowed multiple motivational categories.

\section{Psoriasis and tattoos}

Psoriasis on tattoos was reported in more than a quarter of the patients $(27.6 \%, 13 / 47)$. The delay of onset after tattooing was available for 12 of them and ranged from 1 week to 15 to 20 years after.

No difference was found in terms of psoriasis phenotype when comparing the phenotypes of patients with KP on tattoos with those without (Table 4). Patients with psoriasis on their tattoos reported statistically more tattoos than those without $(p=0.001)$.

Four patients (30\%, 4/12; 8.5\% of the entire series) reported an "early" onset, defined as the rapid development of psoriatic lesions within weeks after tattooing on the tattoos (1 to 3 weeks), 
whereas the eight others reported a "delayed" onset from months to years after, usually 1 to 3 years. The author specifically compared the data between those with an "early" onset of psoriasis and those with a "late" onset (supplemental data). Interestingly, three patients with a rapid onset $(75 \%)$ had a history of KP compared to none among those with a "delayed" onset. It was also observed that those with a "late" onset had considerably more tattoos $(n=6.1)$ than those with an "early" onset $(n=2.5)$. Due to the small sample size, it was not possible to define a psoriasis phenotype at risk among the group of patients. Apart from KP, those with "early" psoriasis had more nail psoriasis (75\%) compared to those with delayed psoriasis $(44 \%)$ or no psoriasis on tattoos at all (26\%).

Psoriasis was restricted to the tattoo of only one respondent, $7.6 \%(1 / 13)$. This patient had only one tattoo thus far. For the patients that had several tattoos (11 of them), none had psoriasis on other tattoos at the same time. If the psoriasis underwent a flareup, two of them $(15 \%, 2 / 13)$ reported that their tattoos were also always affected.

Three patients reported a psoriasis flare-up on another part of the body after tattooing 6.8\% (3/44). No psoriatic arthritis flare-up was reported after tattooing. No patient mentioned that the tattoo had triggered or revealed the psoriasis. One patient reported other adverse events after tattooing, such as local infection, psoriasis, and an allergy to an ink.

Finally, 82\% (37/45) felt that their tattoo(s) had a positive effect on their body image.

Table 4 | Characteristics of the tattooed patients by whether they have psoriasis on their tattoos.

\begin{tabular}{|c|c|c|c|c|}
\hline Characteristic & $\begin{array}{c}\text { Total } \\
\%(n=47)\end{array}$ & $\begin{array}{c}\text { Psoriasis } \\
\text { on tattoos } \\
28 \%(n=13)\end{array}$ & $\begin{array}{c}\text { No psoriasis } \\
\text { on tattoos } \\
72 \%(n=34)\end{array}$ & $p<0.05$ \\
\hline Mean age (SD) & $37.9(9.0)$ & $37.6(9.1)$ & $38.1(9.2)$ & NS \\
\hline Age at onset (SD) & $20.7(9.4)$ & $19.4(8.6)$ & $21.3(9.8)$ & NS \\
\hline $\begin{array}{l}\text { Mean number of } \\
\text { tattoos (SD) }\end{array}$ & $3.0(3.26)$ & $5(5.3)$ & $2.26(1.6)$ & 0.001 \\
\hline Koebner & $21(10)$ & $23(3)$ & $20(7)$ & NS \\
\hline Plaques & $55(26)$ & $61.5(8)$ & $53(18)$ & NS \\
\hline Guttata & $36(17)$ & $46(6)$ & $32(11)$ & NS \\
\hline Pustular & $0(0)$ & - & - & - \\
\hline Folds & $17(8)$ & $15(2)$ & $17(6)$ & NS \\
\hline Nail & $34(16)$ & $54(7)$ & $26(9)$ & NS \\
\hline Scalp & $64(30)$ & $85(11)$ & 56 (19) & NS* \\
\hline Elbows/knees & $42.5(20)$ & $54(7)$ & 38 (13) & NS \\
\hline $\begin{array}{l}\text { Palmo-plantar } \\
\text { pustulosis }\end{array}$ & $8.5(4)$ & $0(0)$ & $12(4)$ & - \\
\hline Psoriatic arthritis & $30(14)$ & $31(4)$ & $30(10)$ & NS \\
\hline
\end{tabular}

\section{Discussion}

This article reports the first and largest study regarding tattooing in a group of patients with psoriasis. Because tattooing causes epidermal damage during the process, some patients with psoriasis are at risk of KP. The number of case reports that has been reported since 1940 is rather low (6 to 20), despite the millions of individuals tattooed worldwide. The difficulty in estimating this complication may be explained as follows. On the one hand, it may be underestimated. Patients may not seek consultation and may treat themselves with local ointments. On the other hand, some patients may be reluctant to get a tattoo because of the fear of a psoriasis flare-up. In this study's series, a third of the patients that did not want a tattoo gave such a reason. Furthermore, there are several clinical phenotypes of psoriasis rather than one, as recently shown by Guinot et al. (26), and KP does not express with the same frequency across these (26). Other factors come into play, such as the extent and activity of psoriasis at the time of the tattoo $(12,14,20)$. Finally, during their lives, patients may also transfer from a state of Koebner positivity to negativity and vice versa (5). Overall, the real frequency of such complications among this patient group is unknown.

We found that less than one-third (27.6\%) of the patients with psoriasis experienced KP on their tattoos. Among them, only approximately $30 \%$ (4/12) reported an acute flare-up of psoriasis within the first weeks after tattooing. Those patients were more likely to have a history of KP. Those with a "late" onset of KP on tattoos seemed to report rather a coincidental localization of psoriasis lesions on their tattoos in the context of a more generalized flare-up. This is indirectly confirmed by the fact that, in this group, they had more tattoos than the group with an "early" onset. More tattoos implies more skin covered, and therefore an increased likelihood of having a fortuitous patch on a tattoo. This study did not estimate the extent of tattooed body surface. One tattoo could cover a wide area, while several tattoos could cover a small area. Based on a recent study performed at a tattoo convention in Finland, the number of tattoos and the tattooed body surface are positively correlated (27), and men's tattoos cover larger surfaces of skin than women's (27). We recently suggested a classification of psoriasis on tattoos in five subgroups based on the literature according to the delay of onset after tattooing, the past history of psoriasis, and the occurrence of psoriasis only on the tattoo or on the body (20).

We always warn patients with psoriasis of the possibility that a patch of psoriasis can occur on the tattoo during the life of the bearer. Patients can be reassured regarding the risk of psoriasis flare-up after tattooing because less than $7 \%$ reported cutaneous psoriasis. There are only a few cases of psoriasis flare-up after tattooing in the literature (16). No psoriatic arthritis flare-up was reported after tattooing. On the other hand, no patients mentioned any clearing of psoriasis after tattooing.

Altogether, $91.5 \%$ (43/47) of patients did not discuss their wish to get a tattoo with their doctor, but 43.5\% (20/46) warned their tattooist about their psoriasis. Patients may perceive tattooing as harmless and may not see the need for medical advice in advance. They may judge the tattooist as being more knowledgeable. Some patients may be reluctant to openly discuss this with their doctor because of a fear of judgment or of being discouraged from getting a tattoo. Among the four patients receiving immunosuppressive treatment, only one patient, receiving a biologic therapy, sought advice from his doctor before getting a tattoo. In the author's experience, biologics such as adalimumab or infliximab may be associated with side effects such as delayed healing of tattoos, local infection, general signs (such as excessive fatigue after the session), and psoriasis flare-up on the tattoo $(12,24)$. Patients receiving immunosuppressive therapy should discuss this with their physician, and decisions should be made on a case-by-case basis (24). In the case of the patient receiving a biologic here, the tattoo session was uneventful. Further data are needed for better advice to patients on this matter.

The motivations for getting a tattoo are numerous and vary by individual. In this group of patients, the "usual" motivations were found, mainly body embellishment, rites of passage (several mentioned a birthday, etc.), expression of personal experiences, and maintaining a self-identity. Some patients got a tattoo simply "because they wanted one." However, one should not deny the beneficial effects of tattoos on patients with chronic diseases. 
It has long been recognized that tattoo(s) can have a bolstering effect on the ego (28). For some patients, tattoos are a means to reclaim their own body, and in the case of chronic skin conditions to gain control over their appearance. We reported previously the beneficial effects of medical alert tattoos among patients with diabetes type 1 (29). There are other reports of young patients getting tattoos naming their conditions to raise concern about them (30). We have experience with patients with neurofibromatosis, congenital epidermolysis bullosa, or vitiligo that received tattoos despite their condition, either for camouflage purposes and/or for controlling their disease. In this study, several patients expressed such an attempt to gain control over their psoriasis. More than $80 \%$ of patients confirmed a positive effect on their body image. This illustrates the importance of listening to patients and not trying to discourage then from getting a tattoo if they express such a wish, but instead discussing the appropriate timing and conditions for this (23).

This study was based on a self-reported internet survey. The author acknowledges the obvious limitations of the study, which include the small sample size, participant selection bias, recollection bias, and possible misunderstanding of the questions. The overrepresentation of women (more than eight out of 10 respondents) was quite striking here, in contrast to which the Finnish Psoriasis Association's members have a female to male ratio of 1.2. Because the questionnaire was freely accessible on the internet, it cannot be guaranteed that the respondents were all members of the Finnish Psoriasis Association. The patients were not examined and the psoriasis status at the time of tattooing was unknown (PASI score, comorbidities, drug history, etc.). Overall, our findings may not be generalizable to the overall population of patients with psoriasis. The aim of this study was to report on

\section{References}

1. Boehncke WH, Schön MP. Psoriasis. Lancet. 2015;386:983-94.

2. Waisman H. Historical note: Koebner on the isomorphic phenomenon. Arch Dermatol. 1981;117:415.

3. Weiss G, Shemer A, Trau H. The Koebner phenomenon: review of the literature. J Eur Acad Dermatol Venereol. 2002;16:241-8.

4. Boyd AS, Neldner KH. The isomorphic response of Koebner. Int J Dermatol. 1990;29:401-10.

5. Fry L. An atlas of psoriasis. 2nd ed. London: Taylor \& Francis; 2004

6. Beerman H, Lane RA. Tattoo: a survey of some of the literature concerning the medical complications of tattooing. Am J Med Sci. 1954;227:444-64.

7. Punzi L, Rizzi E, Pianon M, Rossini P, Gambari PF. Tattooing-induced psoriasis and psoriatic arthritis. Br J Rheumatol. 1997;36:1133-4.

8. Mohla G, Brodell RT. Koebner phenomenon in psoriasis. A common response to skin trauma. Postgrad Med. 1999;106:39-40.

9. Jacob Cl. Tattoo-associated dermatoses: a case report and review of the literature. Dermatol Surg. 2002;28:962-5.

10. Maccarelli FJ, Shenenberger DW. Koebner's phenomenon. Skin trauma may trigger psoriatic flares. Postgrad Med. 2005;118:45-6.

11. Horner KL, Chien AJ, Edenholm M, Hornung RL. Winnie the Pooh and psoriasis too: an isomorphic response of guttate psoriasis in a tattoo. Pediatr Dermatol. 2007;24:E70-2.

12. Kluger N, Debu A, Girard C, Bessis D, Guillot B. [Psoriasis in a tattoo]. Presse Med. 2009;38:338-9. French.

13. Keimig E, Jackson D. Psoriatic eruption after placement of a red tattoo: a case report and review of the literature. J Am Acad Dermatol. 2009;60:AB46.

14. Smith SF, Feldman SR: Tattoo sites and psoriasis. J Drugs Dermatol. 2011;10:1199-1200.

15. Arias-Santiago S, Espiñeira-Carmona MJ, Aneiros-Fernández J. The Koebner phenomenon: psoriasis in tattoos. CMAJ. 2013;185:585.

16. Orzan OA, Popa LG, Vexler ES, Olaru I, Voiculescu VM, Bumbăcea RS. Tattooinduced psoriasis. J Med Life. 2014;7:65-8. patients' "real experiences." Such a methodology was used in prior studies of tattooed individuals with fairly good participation and results (31). It is a fast and cost-effective method. It makes it possible to reach tattooed individuals and patients that may be reluctant to take part in such a study in other settings such as live interviews, and so on.

\section{Conclusion}

It was found that $8.5 \%$ of tattooed patients developed psoriasis within weeks after tattooing. Those patients experienced KP in their past history and also active psoriasis at the time of tattooing. Seven percent reported a psoriasis flare-up on the body after tattooing. Tattooing does not seem to be associated with excessive side effects in patients with psoriasis. On the other hand, a positive effect on the ego and the body image was reported by $82 \%$ of tattooed patients. Patients with psoriasis need proper advising from their healthcare provider. They should able to discuss their wish to get a tattoo with their physician, but should not be discourage from getting a tattoo, but instead advised on the appropriate time to get one. Based on this first study, a larger multicenter study among a cohort of psoriatic patients followed by dermatologists is now being planned.

\section{Acknowledgements}

The author thanks the Finnish Psoriasis Association (Psoriasisliitto), especially Ilona Ruskoaho, communications manager, for her help in creating the questionnaire and with the study. The author is grateful to all of the patients that took time to participate in this study.
17. Ghorpade A. Tattoo-induced psoriasis. Int J Dermatol. 2015;54:1180-2.

18. Tammaro A, Romano I, Parisella F, Persechino F, Persechino S. A case of Koebner phenomenon in a patient with tattoo to lips. J Cosmet Laser Ther. 2016:1-7.

19. Mitchell LV, Caruana DM, Wylie G. Tattoo trouble. BMJ. 2017;356:i6816.

20. Kluger N, Estève E, Fouéré $\mathrm{S}$, Dupuis-Fourdan F, Jegou MH, Lévy-Rameau C. Tattooing and psoriasis: a case series and review of the literature. Int J Dermatol. Forthcoming.

21. Kluger N. Epidemiology of tattoos in industrialized countries. Curr Probl Dermatol. 2015;48:6-20.

22. Myllyniemi S. Taidekohtia. Nuorisobarometri 2009. Helsinki: Yliopistopaino Oy; 2009 [cited 2017 May 11]. 205 p. Available from: https://tietoanuorista.fi/wpcontent/uploads/2013/05/Nourisobarometri_2009.pdf.

23. Kluger N. Contraindications for tattooing. Curr Probl Dermatol. 2015;48:76-87.

24. Kluger $\mathrm{N}$. Tattooing and piercing: an underestimated issue for immunocompromised patients? Presse Med. 2013;42:791-4.

25. Wohlrab S, Stahl J, Kappeler PM. Modifying the body: motivations for getting tattooed and pierced. Body Image. 2007;4:87-95.

26. Guinot C, Latreille J, Perrussel M, Doss N, Dubertret L; French Psoriasis Group. Psoriasis: characterization of six different clinical phenotypes. Exp Dermatol. 2009;18:712-9.

27. Kluger N. Tattooing in Finland: a survey in a tattoo convention. Forum for Nord Derm Ven. 2016;21:7-11

28. Raspa RF, Cusack J. Psychiatric implications of tattoos. Am Fam Physician. 1990;41:1481-6.

29. Kluger N, Aldasouqi S. The motivations and benefits of medical alert tattoos in patients with diabetes. Endocr Pract. 2013;19:373-6.

30. Whittaker RG, Turnbull DM. A diagnostic tattoo. Clin Genet. 2009;75:37-8.

31. Kluger N. Self-reported tattoo reactions in a cohort of 448 French tattooists. Int J Dermatol. 2016;55:764-8. 